\title{
Introduction to Business Value of Smart Devices on the Internet of Things Mini-track
}

\author{
Frederick J. Riggins \\ College of Business \\ North Dakota State University \\ Fargo, ND 58108 \\ fred.riggins@ndsu.edu
}

\author{
Samuel Fosso Wamba \\ Toulouse Business School \\ 31068 Toulouse, France \\ s.fosso-wamba@tbs-education.fr
}

This mini-track addresses issues organizations face as they seek to create and realize business value from incorporating the emerging Internet of Things (IoT) into their organizational infrastructure, their electronic business partner relationships, and the products and services they offer to customers. The IoT is allowing the possibility of tracking and tracing any tagged mobile object as it moves through the value chain thus producing unprecedented end-to-end supply chain visibility. This creates tremendous opportunities for operational and strategic benefits. However, the effective management of this new visibility for improved decision making requires the combination and analysis of data from item-level identification using RFID, sensors, satellites, social media feeds, photos, video and cell phone GPS signals; in short, big data analytics. While the IoT, combined with wireless sensor networks and big data analytics have tremendous potential for transforming various industries, many scholars and practitioners struggle to understand these concepts and capture business value of smart devices being connected through the IoT.

In our first paper entitled "Building Dynamic Capabilities with the Internet of Things," Mary Dunaway, Yulia Sullivan, and Samuel Fosso Wamba propose a useful framework where a firm's dynamic capabilities impact the firm's competitive advantage. In this framework, firms can possess IoT capabilities that allow them to sense and shape opportunities and threats in the competitive environment, better seize upon these opportunities, and finally are able to reconfigure assets and resources for the changing competitive landscape. Using an online questionnaire, they measured 184 respondents to validate their model. This study provides useful measures for IoT capabilities that provide theoretical and practical insights.

Our second paper by Henk Akkermans, Quan Zhu, Feng Fang, Laurens Lamper, and Roland van de Kerkhof entitled "Designing Smart Services: A System
Dynamics-Based Business Modeling Method for IoTEnabled Maintenance Services" outlines a methodology to support decision-making for the introduction of smart maintenance services. The paper describes servitization, smart maintenance services, and the method for modeling potential IoT-based services. The method is illustrated using a case study of a semiconductor equipment OEM.

In our third paper entitled "Enhancing the Building Information Modeling Lifecycle of Complex Structures with IoT: Phases, Capabilities and Use Cases," authors Larissa Gebken, Paul Drews, and Ingrid Schirmer present an IoT capabilities map for Building Information Modeling (BIM). They provide a mapping of BIM phases and capabilities for an overview of use cases in the rail construction sector. This approach provides a blueprint for companies in many industries that seek to embed IoT smart devices in their processes. An important contribution of this paper is a detailed categorization and literature review of IoT use cases mapped to different phases of the BIM lifecycle.

In the last paper of the mini-track, "Design with Perfect Sense: The Adoption of Smart Sensor Technologies in Architectural Practice," Maryam Abhari and Kaveh Abhari examine how architects and design professional can utilize smart sensor technologies in their practice. The authors conducted exploratory interviews with 29 architects and design professionals to examine their attitudes toward adopting IoT technology. They identify a number of influencing factors that correspond to the low adoption rate of smart sensor technology in architectural practice. They categorize these factors as being organizational barriers, environmental (external) barriers, and technological barriers. Specifically, the six factors are perceived risk, perceived value, commitment to learn about the technology, commitment to collaborate with others, and two antecedents that include initial knowledge of the technology and trust. 